03

\title{
Прохождение разрядного тока через границу плазма-электрод в канале электромагнитного рельсового ускорителя
}

\author{
(С) Б.Г. Жуков, Б.И. Резников, Р.О. Куракин, С.А. Поняев, С.В. Бобашев \\ Физико-технический институт им. А.Ф. Иофрфе РАН, \\ 194021 Санкт-Петербург, Россия \\ ฯe-mail: boris.reznikov@mail.ioffe.ru
}

(Поступило в Редакцию 24 марта 2016 г.)

Исследованы явления, сопровождающие ускорение свободного (без ударника) плазменного поршня (ПП) в канале электромагнитного рельсового ускорителя, заполненного различными газами (аргон, гелий). При сильных ударных волнах, создающих за фронтом высокую концентрацию электронов $\sim 10^{17}-10^{18} \mathrm{~cm}^{-3}$, в ударно-сжатом слое возникает интенсивное свечение. Предложен механизм - взрывная электронная эмиссия (ВЭЭ), обеспечивающий высокую эмиссию электронов, протекание части разрядного тока и свечение ударно-сжатого слоя. Определена скорость ударной волны, при которой сильное электрическое поле в дебаевском слое у катода вызывает ВЭЭ его поверхности и прохождение тока в ударно-сжатом слое. Сделан вывод, что при больших скоростях движения плазмы ВЭЭ - универсальный механизм, обеспечивающий прохождение большого тока через границу холодный электрод-плазма.

\section{Введение}

Электромагнитные рельсовые метатели (рельсотроны) применяются в различных областях технической физики как ускорители плазмы и твердых тел. Физической основой метода является ускорение проводника с током в магнитном поле электрической цепи, состоящей из источника питания, двух проводящих параллельных рельсов-электродов и замыкающего рельсы проводника или плазменной перемычки. Плазма инициируется электрическим взрывом проволочки или высоковольтным пробоем межэлектродного промежутка. В этой конфигурации рельсотрон с плазменным поршнем (ПП) может использоваться для ускорения диэлектрических тел, а в отсутствие ударника - для получения высокоскоростных потоков плотной низкотемпературной плазмы. Последние имеют разнообразные применения в современных плазменных технологиях - модификация свойств поверхности плазменными струями [1], физика мощных импульсных МГД устройств [2,3], создание областей высоких плотностей энергии при разгоне и соударении с мишенью сверхзвуковых плазменных струй [4] и т.д.

Эксперименты по ускорению свободного (без ударника) ПП в канале рельсотрона показали, что разгон ПП в каналах, заполненных газами, сопровождается генерацией сильных ударных волн (УВ) [5,6] с параметрами, значительно превышающими значения, достигнутые в традиционных ударных трубах [7]. Существенной особенностью ускорения свободного ПП в рельсотроне является наличие в зоне формирования УВ и движения слоя ударно-сжатого газа достаточно сильного электрического поля $\bar{E}=U_{p p} / w \sim 200-300 \mathrm{~V} / \mathrm{cm}$, где $U_{p p}=100-150 \mathrm{~V}$ омическое падение напряжения на ПП, $w$ - межрельсовое расстояние. При больших скоростях УВ это поле определяет появление новых эффектов, связанных с развитием ионизации газа за УВ $[5,6]$.
При скоростях УВ в аргоне, вызывающих заметную ионизацию газа в ударно-сжатом слое (УСС), в области между УВ и ПП возникает интенсивное свечение газа, сравнимое с интенсивностью свечения ПП, в головной части которого протекает основной ток [5]. Высокая яркость области УСС может быть связана с дополнительным разогревом плазмы из-за перетекания в эту зону части разрядного тока, средняя плотность которого при токах $1-10 \mathrm{kA}$ составляет $6 \cdot\left(10^{6}-10^{7}\right) \mathrm{A} / \mathrm{m}^{2}[6]$. Такая плотность эмиссионного тока соответствует разогреву поверхности медного электрода до температур 3500-4200 K [8], что заметно превышает температуры плавления и кипения меди при нормальном давлении. Визуальный контроль электродов после пуска не выявляет следов плавления их поверхности (см. ниже). Более того, при высоких скоростях УВ $(D \sim 5-10 \mathrm{~km} / \mathrm{s})$ время нагрева электрода плазмой УСС $t_{h}=\Delta / D \sim 3-6 \mu \mathrm{s}$ недостаточно для разогрева области контакта плазмы с электродом протяженностью $\Delta$ до температур, обеспечивающих необходимую плотность тока термоэмиссии. Несогласованность эмиссионного и разрядного токов, определенная в работе [9] как эмиссионный кризис, заставляет искать другой механизм прохождения тока через границу холодный электрод-плазма, не требующий столь высокого разогрева поверхности и обеспечивающий согласование плотностей эмиссионного и разрядного токов.

Единственным известным механизмом, способным обеспечить прохождение большого тока через границу холодный электрод-плазма, является взрывная электронная эмиссия (ВЭЭ) с поверхности электродов в сильных приэлектродных полях, которые возникают при больших скоростях УВ. В этом случае взаимодействие плотной ударно-сжатой плазмы с микронеоднородностями на катоде или диэлектрическими пленками на его поверхности неизбежно приводит к инициированию ВЭЭ. Естественно предположить, что это явление определяет 
прохождение тока в УСС и возникновение свечения в нем, и применить результаты исследований ВЭЭ, представленные в монографиях и статьях, например [10-13]. Целью настоящей работы является интерпретация результатов, полученных в работах $[5,6]$, и детальное обоснование положений эксперимента, основанных на механизме ВЭЭ.

\section{1. Описание эксперимента}

Рельсовый ускоритель и экспериментальные методики описаны в работах [5,6]. Эксперименты проводились при заполнении рельсового канала (длиной $250 \mathrm{~mm}$ ) гелием или аргоном с начальными давлениями $p_{1}=25-500$ Torr. Амплитуда трапецеидального токового импульса с плоской вершиной варьировалась в диапазоне $I_{\max }=10-60 \mathrm{kA}$ изменением начального напряжения на конденсаторах LC-линии в диапазоне $U_{0}=1-4 \mathrm{kV}$. Скорости ПП и УВ нарастали примерно до середины канала, в дальнейшем сохраняя почти постоянное значение вплоть до достижения дульного среза. Во время разряда полярность электродов не изменялась. Выбор для исследований аргона и гелия был связан с тем, что при низких скоростях УВ термодинамические параметры аргона и гелия весьма близки, а при начальном давлении аргона, в 10 раз меньшем начального давления гелия, их начальные плотности $\rho_{1}$ равны. В этом случае зависимости скорости УВ от напряжения $U_{0}$ в каналах, заполненных аргоном и гелием, практически совпадали [6]. Наиболее существенное отличие исследуемых газов в значениях потенциалов ионизации (аргон $-15.76 \mathrm{eV}$, гелий $-24.59 \mathrm{eV})$ проявляется при генерации ионизующих УВ. В этом случае концентрация электронов за УВ в аргоне на несколько

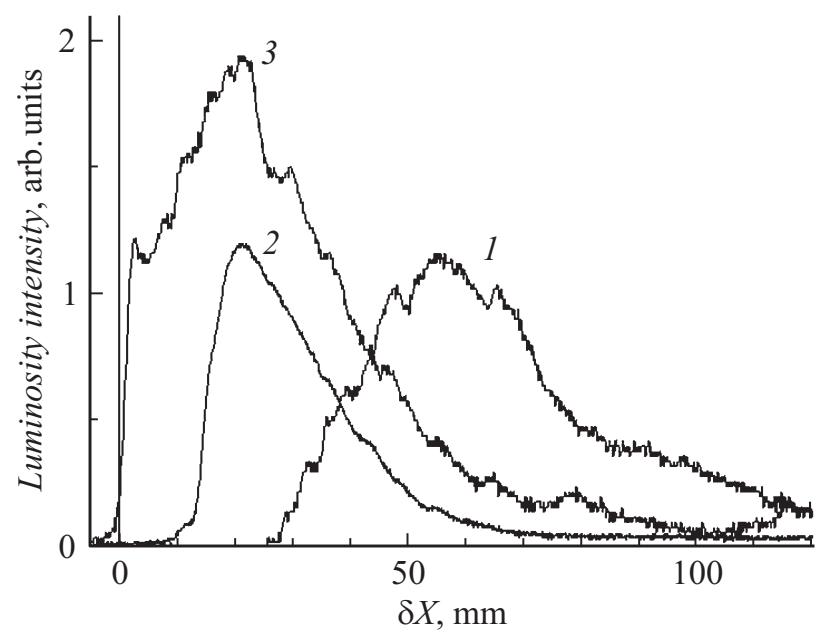

Рис. 1. Распределение интенсивности свечения в ударносжатом слое и плазменном поршне; $\delta x=\left(t-t_{0}\right) D-$ расстояние от ударной волны, $t_{0}-$ момент прохода контрольного сечения ударной волной. $1-\mathrm{He}, D=7.3 \mathrm{~km} / \mathrm{s}, p_{1}=500$ Torr, $U_{0}=2.2 \mathrm{kV} ; 2-\mathrm{Ar}, D=4 \mathrm{~km} / \mathrm{s}, p_{1}=100$ Torr, $U_{0}=1.2 \mathrm{kV}$; $3-\operatorname{Ar}, D=7 \mathrm{~km} / \mathrm{s}, p_{1}=50$ Torr, $U_{0}=2.2 \mathrm{kV}$. порядков выше, чем в гелии, что, как показал эксперимент, существенно меняет картину течения ударносжатого аргона из-за наличия в канале электрического поля $(E=200-300 \mathrm{~V} / \mathrm{cm})$.

На рис. 1 представлено распределение интенсивности свечения УСС вдоль канала, в зависимости от координаты сечения, примерно равной его расстоянию от УВ. Здесь $t_{0}$ - время прохождения сечения канала, находящегося на расстоянии $45 \mathrm{~mm}$ от выхода. Такое представление экспериментальных результатов позволяет сравнивать распределения яркости в зоне свечения для различных режимов. Все осциллограммы, показанные на рис. 1, сняты при одинаковых коэффициентах усиления регистрирующего тракта. При заполнении канала гелием свечение УСС между фронтом УВ и ПП не наблюдалось во всем исследованном диапазоне скоростей, верхняя граница которого равнялась максимальной скорости УВ $16.4 \mathrm{~km} / \mathrm{s}$, полученной в канале при начальном давлении 25 Torr. Аналогичная картина имела место и для аргона при скорости УВ не выше $D \sim 4-5 \mathrm{~km} / \mathrm{s}$. Однако, при бо́льших скоростях УВ интенсивное свечение в аргоне возникало сразу за фронтом УВ и по яркости было сопоставимо с излучением от ПП.

\section{2. Развитие ВЭЭ в канале рельсотрона}

Рассмотрим изменение условий в канале рельсотрона при увеличении скорости УВ. При малых скоростях УВ концентрация заряженных частиц за УВ пренебрежимо мала, и ток в ударно-сжатом слое отсутствует. По мере увеличения скорости УВ газ, прошедший сжатие в УВ, разогревается, ионизуется, и в объеме ударно-сжатого слоя создается все возрастающая плотность заряженных частиц. В отсутствие электрического тока под действием электрического поля в канале в ударно-сжатом слое вблизи катода образуется слой положительного объемного заряда толщиной порядка дебаевского радиуса $r_{d}=\left[8 \pi e^{2} n_{e} /(k T)\right]^{-1 / 2}$. Значительная часть омического падения напряжения между рельсами сосредоточивается на этом слое $U \approx(0.8-0.9) U_{p p}$. В результате в дебаевском слое возникает электрическое поле $E \simeq U / r_{d}$, вызывающее автоэлектронную эмиссию с поверхности катода. С ростом скорости УВ величина этого поля также растет

$$
E(D)=0.205 U\left(n_{e} / T\right)^{1 / 2}[\mathrm{~V} / \mathrm{cm}],
$$

так как определяется концентрацией электронов в ударном слое $n_{e}$ и температурой $T$. Последние являются функциями скорости УВ $D$ и начального давления газа в канале.

Электрическое поле у поверхности катода снижает высоту потенциального барьера на границе металлплазма. Это приводит к резкому увеличению скорости нарастания тока автоэлектронной эмиссии и омическому нагреву катода, где максимальная тепловая энергия выделяется вблизи микронеоднородностей его поверхности. Здесь происходит локальное увеличение поля 
и эмиссионного тока, что после накопления энергии за время $t_{\exp }$ приводит к тепловому взрыву нагреваемого объема. При достижении током автоэмиссии пороговых значений $\sim 10^{8}-10^{9} \mathrm{~A} / \mathrm{cm}^{2}$ время задержки взрыва локальной области $t_{\exp }$ порядка нескольких наносекунд [11], что намного меньше характерного времени изменения скорости УВ $t_{v} \sim 1 \mu \mathrm{s}$. В этом случае электрическое поле и эмиссионный ток становятся функциями текущего значения скорости УВ.

После взрыва области поверхности с электрода истекает струя плотной $\left(n_{e} \sim 10^{20} \mathrm{~cm}^{-3}\right)$ плазмы (катодный факел) с начальной скоростью $\sim 10^{6} \mathrm{~cm} / \mathrm{s}$. При выбросе за пределы дебаевского слоя катодных факелов, состоящих из плазмы, паров материала и десорбированных с катода газов, устанавливается электрический контакт между холодным катодом и плазмой ударно-сжатого слоя. Последняя имеет высокие значения температуры $(1-2) \cdot 10^{4} \mathrm{~K}$, давления $10-50 \mathrm{~atm}$ и удельной электропроводности $\sigma=50 \mathrm{~S} / \mathrm{cm}$, которая сравнима с еe значением в ПП [6]. На отрицательном электроде происходит постоянное возобновление микровзрывов, которые инициируются электрическим контактом с ударносжатой плазмой. На этой стадии происходит переход от взрывной эмиссии к дуговому разряду, когда за счет эмиссии электронов из внешних слоев объема нагретой плазмы с тепловой скоростью $\bar{v}_{H}$ устанавливается электрический контакт с холодным анодом. Плотность тока $j=e n_{e} \bar{v}_{H}$, эмитированного плазмой на анод, для условий аргоновой плазмы, сжатой УВ, движущейся со скоростью $4.5-5 \mathrm{~km} / \mathrm{s}$, составляет $15-28 \mathrm{kA} / \mathrm{cm}^{2}$, что обеспечивает начальный ток и прохождение тока во всем объеме ударно-сжатого слоя через образовавшуюся параллельную электрическую цепь.

\section{3. Условие возникновения ВЭЭ, ток эмиссии}

В разд. 3 рассчитаны зависимости электрического поля и эмиссионного тока от скорости УВ и определено пороговое значение скорости УВ, при превышении которого в ударно-сжатом слое возникает свечение газа.

Выражение для плотности тока автоэлектронной эмиссии $j$, полученное Фаулером и Нордгеймом, описывает его зависимость от напряженности электрического поля $E$ вблизи поверхности металла [8], которое во всех формулах имеет размерность $\mathrm{V} / \mathrm{cm}$

$$
\begin{aligned}
j\left[\mathrm{~A} / \mathrm{cm}^{2}\right]= & 6.2 \cdot 10^{-6} \frac{\left(\varepsilon_{0} / \varphi\right)^{1 / 2} E^{2}}{\varepsilon_{0}+\varphi} \\
& \times \exp \left(-\frac{6.85 \cdot 10^{7} \varphi^{3 / 2} \xi(\Delta \varphi / \varphi)}{E}\right) .
\end{aligned}
$$

Выражение (2) не учитывает разогрев или охлаждение катода при автоэлектронной эмиссии (эффект Нотингама) [11], однако оно вполне пригодно для нашего анализа. Здесь $\varepsilon_{0}$ - граничная энергия Ферми, $\varphi$ и $\Delta \varphi(E)$ - работа выхода при $E=0$ и ее снижение во внешнем поле, $\xi(\Delta \varphi / \varphi)$ - поправочный множитель, изменяющийся с увеличением поля в интервале $[0,1]$. Для меди $\varepsilon_{0}=7 \mathrm{~V}, \varphi=4.4 \mathrm{~V}$. Снижение работы выхода (эффект Шоттки) дается выражением [8]

$$
\Delta \varphi=3.8 \cdot 10^{-4} E^{1 / 2}[\mathrm{~V}] .
$$

Из соотношений (1), (3) и (2) следует, что электрическое поле, снижение работы выхода и ток эмиссии возрастающие функции скорости УВ.

Из соотношения (3) можно оценить характерные величины, ограничивающие сверху максимальные значения электрического поля и эмиссионного тока. В частности, поле $E_{*}=(\varphi / 3.8)^{2} \cdot 10^{8} \mathrm{~V} / \mathrm{cm}=1.34 \cdot 10^{8} \mathrm{~V} / \mathrm{cm}$ coответствует нулевому значению работы выхода $\Delta \varphi=\varphi$ и значению эмиссионного тока $j_{*}=12.3 \cdot 10^{9} \mathrm{~A} / \mathrm{cm}^{2}$, что на порядок превышает пороговые значения, при которых происходит электрический взрыв поверхности [11]. Это ограничивает сверху диапазон скоростей УВ, в которых можно использовать выражения (1)-(3), условием $D<D_{\max }$, при этом скорость $D_{\max }$ определяется условиями $\delta \varphi_{\max }=(\Delta \varphi / \varphi)_{\max } \leq 0.9$ или $j \leq j_{\max }=10^{9} \mathrm{~A} / \mathrm{cm}^{2}$.

Параметры газа за УВ, необходимые для расчета поля (1) и эмиссионного тока (2), определялись из решения системы уравнений сохранения массы, импульса и энергии на разрыве, дополненными термическим и калорическим уравнениями состояния плазмы и уравнениями, описывающими равновесную ионизацию атомов в неидеальной плазме [6].

Достаточно простую оценку для скорости УВ $D_{t h}$, при которой происходит электрический взрыв поверхности катода, протекание тока в ударно-сжатом слое и возникновение свечения, можно получить, предполагая, что вблизи поверхности электрическое поле $E=U / r_{d}$. С учетом выражений (1) и (3) можно определить концентрацию $n_{e}$ и пороговую скорость УВ $D_{t h}$ как функцию $\delta \varphi_{t h}$ и $U$

$$
F\left(D_{t h}\right)=\left(\frac{n_{e} \cdot 10^{-17}}{T / 10^{4}}\right)^{1 / 4}=C=1.242 \frac{\varphi}{3.8} \frac{\delta \varphi_{t h}}{(U / 100)^{1 / 2}} .
$$

При $\delta \varphi_{t h}=0.9, \quad \varphi=4.4 \mathrm{~V}, \quad U=100 \mathrm{~V} \quad$ постоянная $C \approx 1.3$. Из уравнения (4), используя табличные значения параметров газа за УВ, находим скорость $D_{t h}$. При $p_{1}=25$ Torr в аргоне значение $D_{t h} \approx 4.5 \mathrm{~km} / \mathrm{s}$. Полученное численное значение $D_{t h}$ достаточно близко к значению наименьшей скорости УВ, при которой в ударно-сжатом слое наблюдается свечение. Вполне удовлетворительная точность такой оценки связана с чрезвычайно резкой зависимостью значений $\delta \varphi$ и тока эмиссии от скорости УВ.

\section{4. Результаты расчета}

На рис. 2-4 представлены зависимости от скорости УВ электрического поля в дебаевском слое (рис. 2), тока 
автоэлектронной эмиссии (рис. 3) и уменьшения работы выхода электронов $\delta \varphi$ (рис. 4). Расчет ограничивался максимальным значением скорости $D_{\max }$, соответствующей плотности тока эмиссии $j \leq j_{\max } \approx 1.2 \cdot 10^{9} \mathrm{~A} / \mathrm{cm}^{2}$. Все зависимости демонстрируют взрывной характер увеличения поля и эмиссионного тока от скорости УВ в области параметров, где величина $\delta \varphi \geq 0.5$. Из-за этого значение $D_{t h}$ весьма слабо зависит от величин, входящих в выражение $C$.

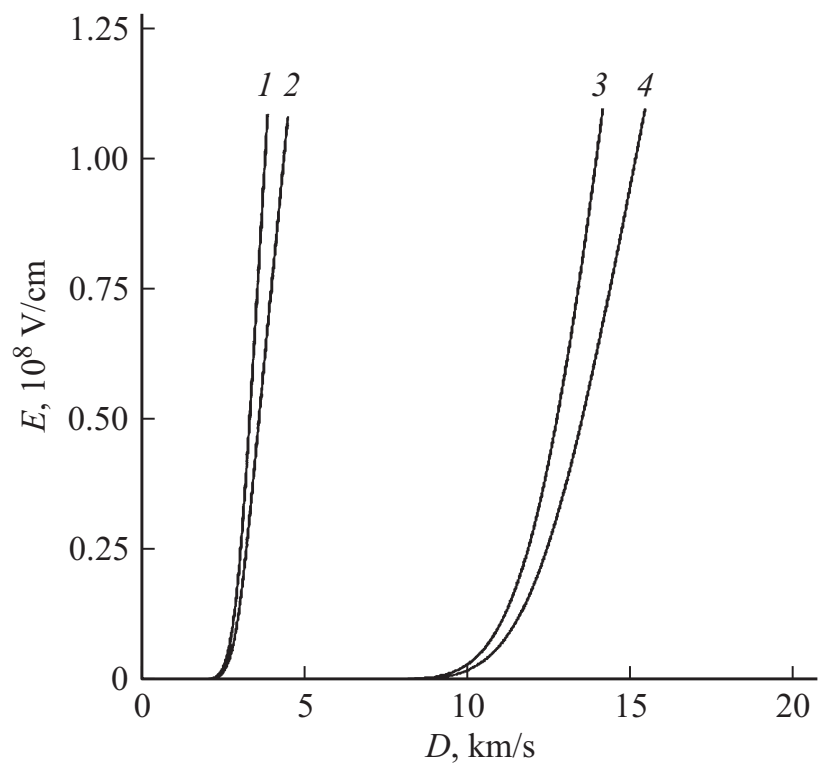

Pис. 2. Электрическое поле в дебаевском слое вблизи катода при сильных УВ в аргоне: $p_{1}=25$ Torr $-1,2$ и в гелии $p_{1}=250$ Torr -3 , 4. Падение напряжения на дебаевском слое $U: 1,3-160 \mathrm{~V}, 2,4-100 \mathrm{~V}$.

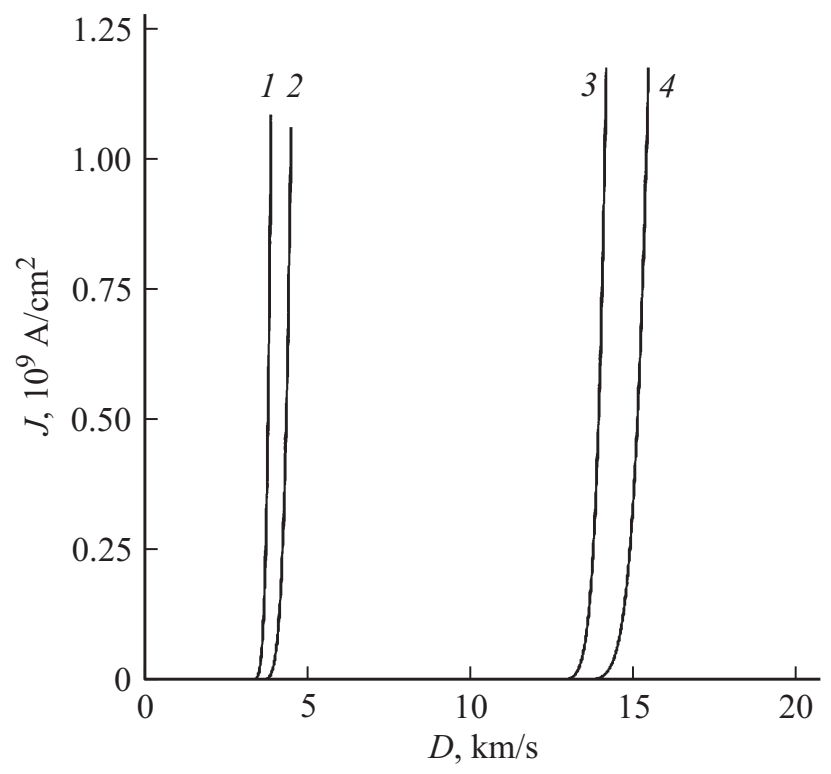

Pис. 3. Ток автоэлектронной эмиссии из медных электродов при сильных УВ в аргоне и в гелии. Нумерация кривых как на рис. 2.

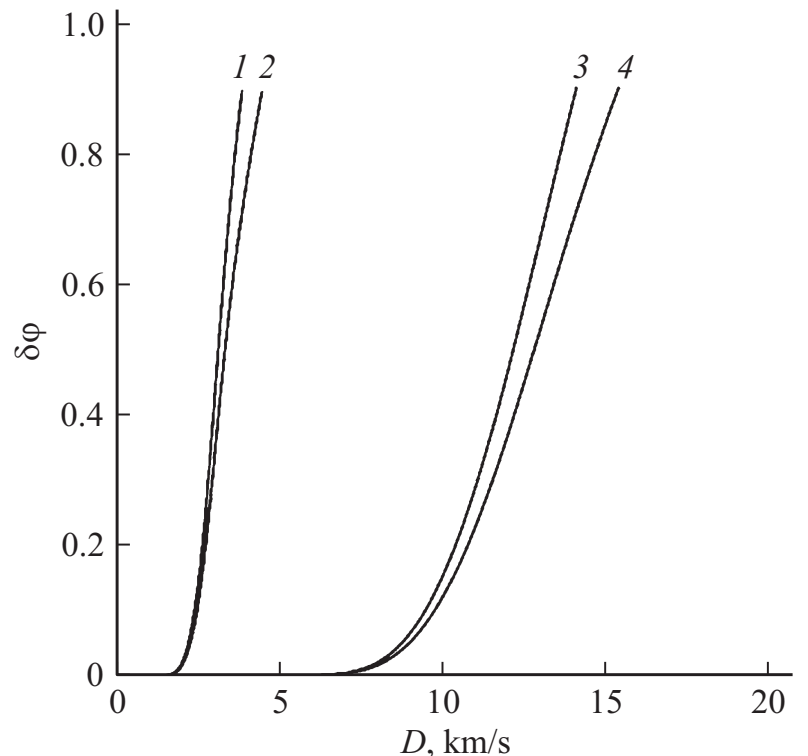

Рис. 4. Снижение работы выхода медных электродов $\delta \varphi=\Delta \varphi / \varphi$ при сильных УВ в аргоне и в гелии. Нумерация кривых как на рис. 2.

Для иллюстрации этих качественных особенностей получим приближенное аналитическое решение уравнения (4) и определим скорость УВ $D_{\text {th }}$, при которой происходит взрывная эмиссия. Плотность электронов $n_{e}$ в единице объема находится из уравнения Саха [14]. При $x_{e}=n_{e} / n \ll 1$

$$
n_{e} \approx \sqrt{K n}, K=2 \frac{u_{1}}{u_{a}}\left(\frac{2 \pi m_{e} k T_{2}}{h_{p}^{2}}\right)^{3 / 2} \exp \left(-\frac{I}{k T_{2}}\right) .
$$

Здесь $n=p_{2} /\left(k T_{2}\right)$ полное число частиц в единице объема, $K-$ константа равновесия реакции ионизации $a \rightleftarrows a^{+}+e, u_{1}, u_{a}-$ статистические суммы электронного возбуждения иона и атома, $m_{e}-$ масса электрона, $h_{p}, k-$ постоянные Планка и Больцмана, $I$ - потенциал ионизации, $p_{2}, T_{2}$ - давление и температура за ударной волной, которые определяются из законов сохранения массы, импульса и энергии на разрыве и являются функциями скорости УВ [14]. Для сильных УВ $\left(p_{2} \gg p_{1}, h_{2} \gg h_{1}\right)$

$$
\begin{gathered}
p_{2}=10^{6} \rho_{1} D^{2}\left(1-\rho_{1} / \rho_{2}\right), \quad T_{2}=h_{2 m} / C_{2 m}, \\
h_{2 m}=10^{6} D^{2} / 2\left[1-\left(\rho_{1} / \rho_{2}\right)^{2}\right], \quad C_{p m}=10^{3}(R / \mu) \gamma /(\gamma-1) .
\end{gathered}
$$

Здесь $\rho_{1}, \rho_{2}-$ плотность газа до и за УВ, $D$ в единицах $\mathrm{km} / \mathrm{s}, h_{2 m}$ - удельная энтальпия на единицу массы в $\mathrm{J} / \mathrm{kg}, C_{p m}$ и $\gamma$ - эффективные значения теплоемкости на единицу массы и показателя адиабаты, $R=8.314 \mathrm{~J} /(\mathrm{K} \cdot \mathrm{mol})$ - универсальная газовая постоянная, $\mu=\mu_{a}\left(1-x_{e}\right)$ - молярная масса газа в g/mol. C использованием соотношений (5) получаем выражение для 
функции $F(D)$

$$
\begin{gathered}
F(D)=\left(\frac{n_{e} \cdot 10^{-17}}{T_{2} \cdot 10^{-4}}\right)^{1 / 4}=A^{1 / 4} \exp \left(-\frac{I}{8 k T_{2}}\right)\left(\frac{p_{2}}{T_{2}^{3 / 2}}\right)^{1 / 8}, \\
A=10^{-13}\left[2 \frac{u_{1}}{u_{a}}\left(\frac{2 \pi m_{e}}{h_{p}^{2}}\right)^{3 / 2} k^{1 / 2}\right]^{1 / 2}, \\
\left(\frac{p_{2}}{T_{2}^{3 / 2}}\right)^{1 / 8}=\frac{C_{1}}{D^{1 / 8}}, C_{1}=\frac{\left[\rho_{1}\left(1-\rho_{1} / \rho_{2}\right)\right]^{1 / 8} 10^{1.25}}{[500 \mu(\gamma-1) /(R \gamma)]^{3 / 16}} .
\end{gathered}
$$

Из уравнения (4) $F(D)=C$ и выражений (7), (8) находим температуру газа за УВ как функцию $D=D_{t h}$

$$
T_{2}=T_{*} / \ln \left(\frac{B}{D^{1 / 8}}\right), T_{*}=\frac{I}{8 k}, B=A^{1 / 4} C_{1} / C .
$$

Это выражение с учетом соотношений (6) приводится к слабо нелинейному уравнению для вычисления пороговой скорости, которая зависит от двух постоянных $D_{*}$ и $B$

$$
D=D_{*} / \sqrt{\ln \frac{B}{D^{1 / 8}}}, D_{*}=\sqrt{2 C_{p m} T_{*}} .
$$

Постоянные $A$ и $C_{1}(8)$ вместе с величинами $T_{*}, D_{*}, B$ характеризуют преобразование кинетической энергии УВ во внутреннюю энергию плазмы при сжатии, нагреве и частичной ионизации газа УВ. Постоянная $C$ (4) описывает связь электрического поля и снижение высоты потенциального барьера на границе металлплазма от плотности объемного заряда $n_{e}$ в дебаевском слое вблизи электродов. Отметим, что величина $C_{1} \sim \rho_{1}^{1 / 8} \mu^{-3 / 16} \quad$ (8) слабо зависит от начального давления в канале $p_{1}$ и молярной массы газа $\mu$. При вычислении $C_{p m}$ и $C_{1}$ размерности $[\mu]=\mathrm{g} / \mathrm{mol}$, $\left[\rho_{1}\right]=\mathrm{g} / \mathrm{cm}^{-3}$, а размерность скорости УВ $\mathrm{km} / \mathrm{s}$. Значения постоянных $A^{1 / 4}=5.864, C=1.294$, а $C_{1} \approx 1.53$ (для рассматриваемого случая ускорения ПП в аргоне при $p_{1}=25$ Torr, $T_{1}=293 \mathrm{~K}$ ). При этом $D_{*}=5.98 \mathrm{~km} / \mathrm{s}$, $B=6.93(\mathrm{~km} / \mathrm{s})^{1 / 8}$. Решение уравнения (10) имеет наибольшую чувствительность к значению $D_{*}$, которое зависит от потенциала ионизации газа.

Уравнение (10) легко решается методом итераций. Подстановка в правую часть $D=D_{*}$ дает отличие менее $1 \%$ от точного значения решения $D_{t h}=4.52 \mathrm{~km} / \mathrm{s}$, это позволяет рассматривать (10) как определение значения $D_{t h}$. При изменении начального давления аргона на порядок $p_{1}=250$ Torr пороговая скорость УВ уменьшается примерно на $10 \%$, что связано с увеличением плотности заряженных частиц в УСС. При этом ВЭЭ возникает, если длина канала рельсотрона и рабочий ток обеспечивают выполнение условия $D_{\max }>D_{t h}$. Аналогичные простые оценки для начальных давлений гелия $p_{1}=25$ и 250 Torr дают значения $D_{t h}=19.7 \mathrm{~km} / \mathrm{s}$ и $D_{t h}=18.1 \mathrm{~km} / \mathrm{s}$. Значительное превышение пороговой скорости в гелии по сравнению с ее значением в аргоне связано с высоким потенциалом ионизации гелия.

\section{5. Обсуждение результатов и заключение}

Суммируем основные результаты работы. Определен механизм прохождения тока через границу холодный электрод-плазма в электромагнитном рельсовом ускорителе. Это взрывная термоавтоэлектронная эмиссия с поверхности электродов, детально изученная при высоковольтном импульсном разряде в вакууме [10-13]. При высоких скоростях УВ данный механизм обеспечивает протекание тока в ударно-сжатом слое и свечение плазмы УСС между УВ и ПП. При „малых“ рабочих токах и „больших,, начальных давлениях газа максимальная скорость УВ в канале не обеспечивает условий возникновения взрывной эмиссии, и свечение в ударносжатом слое отсутствует [6]. Эти выводы подтверждаются пороговым характером возникновения свечения в ударно-сжатом слое, зависимостью пороговой скорости УВ от состояния поверхности рельсов, влиянием материала электродов на скорость УВ [15] и близостью теоретических и экспериментальных значений пороговой скорости УВ.

Более того, ВЭЭ есть универсальный механизм для прохождения тока через границу плазма-холодный электрод. Она обеспечивает перемещение ПП вдоль канала, где после инициации ПП высоковольтным разрядом и его формирования в дебаевском слое вблизи поверхности катода создается сильное электрическое поле при разделении зарядов в плазме с большой концентрацией заряженных частиц. При смещении плазменной перемычки в новое положение электрический контакт с рельсами переднего фронта движущейся плазмы сначала отсутствует, а затем устанавливается через несколько наносекунд после того, как сильное поле вызывает взрывную эмиссию электронов с поверхности рельсов. На фотографиях поверхности отрицательного электрода, сделанных после однократного запуска (рис. 5,a-c), видна система эрозионных пятен малого размера (микрократеров), более характерных для искровых разрядов. Сплошные протяженные зоны сильной эрозии отсутствуют. Из-за того что разрядный ток в ПП значительно превышает ток через УСС, следы эрозии на поверхности рельса вызваны в основном током, протекающим через ПП.

На фотографиях областей поверхности, сделанных на различных расстояниях $x_{c h}$ от места инициации разряда, видно отсутствие сплошного повреждения поверхности и протяженных зон расплавления, о которых сообщается в [16]. Рисунок поверхности представляет набор отдельных катодных пятен малого размера, состоящих из отдельных ячеек размером 5-6 $\mu \mathrm{m}$. Это означает, что распределение тока разряда неравномерно, и разрядный ток сосредоточен в токовых каналах. По внешнему виду пятна, согласно принятой классификации в $[10,13]$, относятся к пятнам второго типа и образуются при взрывах микроскопических выступов на поверхности электрода при протекании ВЭЭ. 

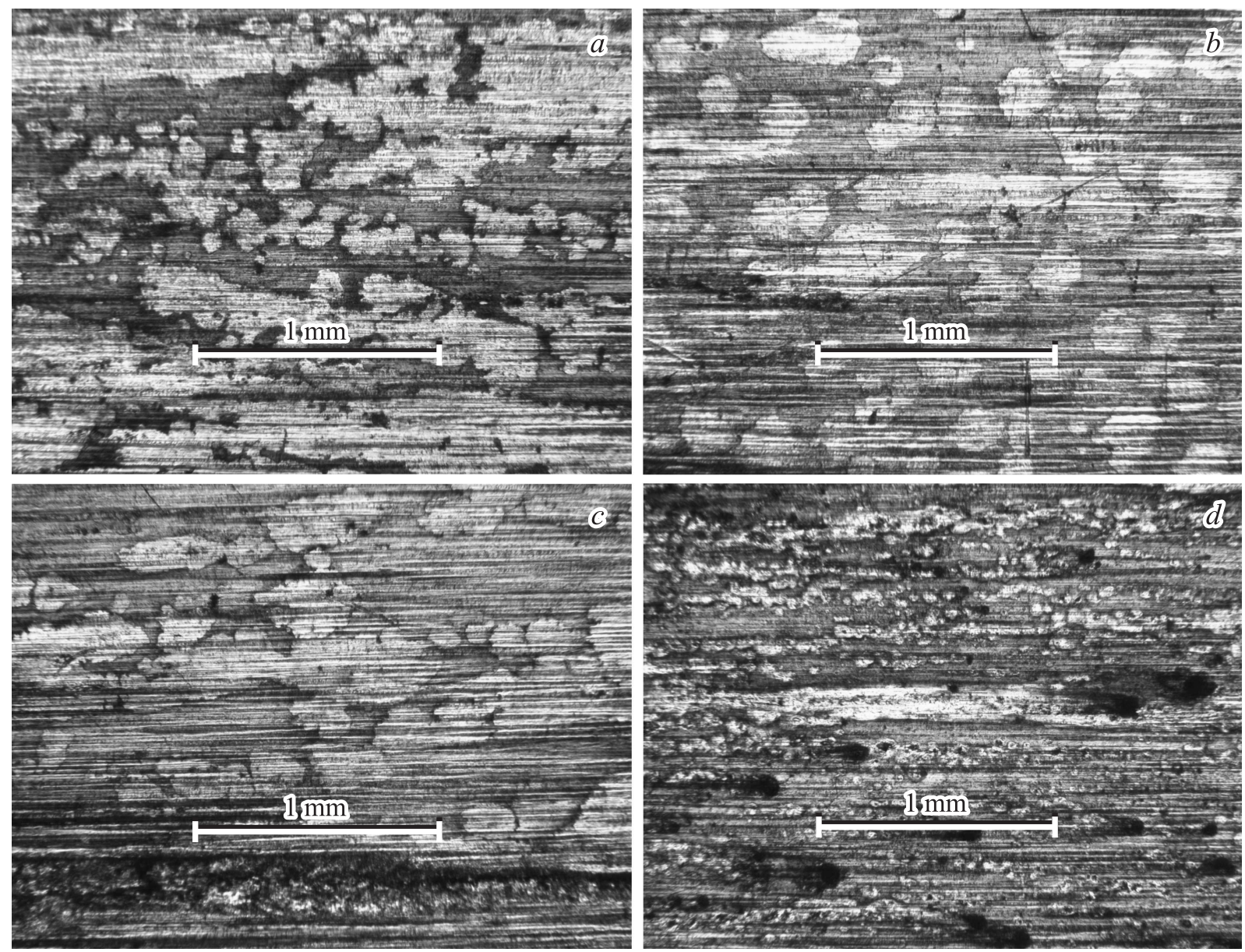

Рис. 5. Фотографии области поверхности электродов после однократного запуска в аргоне и в гелии при различных начальных давлениях; $a, b, c-$ катод, $d-$ анод, $x_{c h}-$ расстояние от места инициации разряда. $a-\mathrm{Ar}, 25 \mathrm{Torr}, x_{c h}=100 \mathrm{~mm} ; b-\mathrm{Ar}$, 25 Torr, $x_{c h}=30 \mathrm{~mm} ; c-$ Ar, 250 Torr, $x_{c h}=170 \mathrm{~mm} ; d-$ Ar, 25 Torr, $x_{c h}=170 \mathrm{~mm}$.

Типичная фотография поверхности анодного рельса (рис. $5, d)$ показывает, что и здесь ток не распределяется равномерно по поверхности. Это обстоятельство, повидимому, свидетельствует о неустойчивостях (контракции) [10], развивающихся в потоке электронов, эмитируемых на анод из контактирующей с ним плазмы.

Сделанный вывод о роли ВЭЭ, по нашему мнению, решает проблему согласования разрядного и эмиссионного токов в рельсотроне, которая обсуждается уже в течение 25 лет при рассмотрении ускорения ПП диэлектрических ударников $[9,16]$. На начальном участке разгона, когда скорости движения ПП малы и время нагрева электродов значительно больше, не исключается термоэмиссионный механизм прохождения тока.

Роль взрывной электродной эмиссии не сводится только к обеспечению прохождения тока через границу электрод-плазма. Протекание части разрядного тока через проводящий ударно-сжатый слой, вызванное ВЭЭ, влияет на динамику ПП. Во-первых, уменьшается ток в ПП и ускоряющая амперова сила $F_{A}$, во-вторых, изменяется количество эрозионной массы, поступающей в ПП и ударно-сжатый слой. Существенно, что эти явления происходят в режиме насышения скорости ПП, когда суммарная сила, действующая на ПП, много меньше ускоряющей силы $F_{s u m}=F_{A}-F_{d}-F_{e r}$ из-за действия силы давления газа за УВ $F_{d}$ и тормозящей силы $F_{e r}$, возникающей при увлечении ПП эрозионной массы, поступающей в канал с поверхности электродов [6]. Влияние прохождения тока в УСС на динамику и максимальную скорость ПП ранее рассматривалось в рамках упрощенной модели [17]. Этот вопрос требует дальнейших исследований.

\section{Список литературы}

[1] Погребняк А.Д., Тюрин Ю.Н. // УФН. 2005. Т. 175. Вып. 5. C. 515-544.

[2] Взрывные генераторы мощных импульсов электрического тока / Под ред. В.Е. Фортова. М.: Наука, 2002. 399 с.

[3] Асиновский Э.И. и др. / Импульсные МГД-преобразователи химической энергии в электрическую / Под ред. А.Е. Шейндлина, В.Е. Фортова. М.: Энергоатомиздат, 1997. $267 \mathrm{c}$. 
[4] Hsu S.C., Merritt E.C., Moser A.L. et al. // Physics of Plasma. 2012. Vol. 19. P. 123514.

[5] Бобашев С.В., Жуков Б.Г., Куракин Р.О., Поняев С.А., Резников Б.И. // Письма в ЖТФ. 2014. Т. 40. Вып. 22. C. $37-44$.

[6] Бобамев С.В., Жуков Б.Г., Куракин Р.О., Поняев С.А., Резников Б.И., Твердохлебов К.В. // ЖТФ. 2015. Т. 85. Вып. 1. С. 39-46.

[7] Великович А.Л., Либерман М.А. Физика ударных волн в газах и плазме. М.: Наука, 1987. 295 с.

[8] Райзер Ю.П. Физика газового разряда. Долгопрудный, 2009. $734 \mathrm{c}$

[9] Ткаченко Г.В., Урюков Б.А. // ТВТ. 2014. Т. 52. Вып. 5. C. 797.

[10] Месяи Г.А. Взрывная автоэлектронная эмиссия. М.: Физматлит, 2011. $280 \mathrm{c}$.

[11] Королев Ю.Д., Месяи, Г.А. Автоэмиссионные и взрывные процессы в газовом разряде. Новосибирск: Наука, 1982. $285 \mathrm{c}$.

[12] Литвинов У.А., Месяи Г.А., Проскуровский Д.И. // УФН. 1983. Т. 139. Вып. 2. С. 265-302.

[13] Баренгольи С.А., Месяи, Г.А., Цвентух М.М. // ЖЭТФ. 2008. Т. 134. Вып. 6 (12). С. 1213-1224.

[14] Зельдович Я.Б., Райзер Ю.П. Физика ударных волн и высокотемпературных гидродинамических явлений. М.: Наука, 1966. $686 \mathrm{c}$.

[15] Бобашев С.В., Жуков Б.Г., Куракин Р.О., Поняев С.А., Резников Б.И. // Письма в ЖТФ. 2015. Т. 41. Вып. 19. C. 96-104.

[16] Бейлис И.И., Осташев В.Е. // ТВТ. 1989. Т. 27. Вып. 6. C. 1041.

[17] Дьяков Б.Б., Резников Б.И. Материалы I Всесоюз. семинара по динамике сильноточного дугового разряда в магнитном поле. Новосибирск, 10-13 апреля 1990 г. C. $38-68$. 